\title{
Investigation of tolerability and quality of life for carboplatin-based chemotherapy in an elderly urothelial carcinoma patient undergoing hemodialysis: a case report
}

Masahiro Kondo ${ }^{1}$, Taku Naiki ${ }^{2}$, Yuji Hotta ${ }^{3}$, Yuko Yamamoto ${ }^{4}$, Yosuke Sugiyama', Takahiro Yasui ${ }^{2}$ and Kazunori Kimura ${ }^{1,3,5^{*}}$

\begin{abstract}
Background: To our knowledge, no studies have evaluated the safety of carboplatin (CBDCA)-based chemotherapy in hemodialysis patients $>80$ years-old. In addition, the impact of CBDCA-based chemotherapy on such elderly patients' quality of life (QOL) is unknown. We report a case of gemcitabine plus CBDCA chemotherapy treatment in an 81-year-old man with metastatic urothelial carcinoma undergoing hemodialysis.

Case presentation: The optimal CBDCA dose and hemodialysis timing were determined by monitoring the measured area under the concentration-time curve (AUC) of CBDCA. This was used because the AUC of CBDCA is correlated with hematologic toxicities, especially nadir thrombocytopenia, and CBDCA is easily dialyzed during hemodialysis. In the first cycle, a 160 mg CBDCA dose, calculated using Calvert's formula (target-AUC: 5), was administered on day 1. Hemodialysis was performed for $3 \mathrm{~h}$, starting $2 \mathrm{~h}$ after the end of the CBDCA infusion. The measured-AUC was $5.96 \mathrm{mg} / \mathrm{mL}$ min in the first cycle, after which the patient developed grade $3 / 4$ hematologic toxicities. Thus, in the second cycle, the CBDCA dose was reduced to $135 \mathrm{mg}$ and the time interval between CBDCA infusion and hemodialysis was shortened to $1 \mathrm{~h}$, according to the results of a pharmacokinetic study performed using parameters from the first cycle. The measured-AUC in the second cycle was $4.97 \mathrm{mg} / \mathrm{mL}$ min, and hematologic toxic effects decreased to grade 2. Stable disease according to the Response Evaluation Criteria in Solid Tumors was demonstrated after the second and third cycles. QOL scores determined using a short-form questionnaire (SF-36) after 2 cycles were not significantly lower than pretreatment values. Conclusions: CBDCA-based chemotherapy is clinically acceptable in hemodialysis patients aged $>80$ years, and this systemic chemotherapy can be a treatment option in such elderly patients undergoing hemodialysis. However, the measured-AUC should be monitored, as the actual AUC is unpredictable in hemodialysis patients. This is due to the influence of various factors that may be different for each patient, such as the patient's residual renal function and hemodialysis duration and conditions, especially in elderly patients, who have a higher risk of chemotherapy-induced neutropenia.
\end{abstract}

Keywords: Carboplatin, Hemodialysis, Pharmacokinetics, Quality of life, Elderly

\footnotetext{
* Correspondence: kkimura@med.nagoya-cu.ac.jp

'Department of Pharmacy, Nagoya City University Hospital, 1-Kawasumi,

Mizuho-cho, Mizuho-ku, Nagoya, Aichi 467-8602, Japan

${ }^{3}$ Department of Hospital Pharmacy, Graduate School of Pharmaceutical

Sciences, Nagoya City University, 3-1, Tanabe dori, Mizuho ku, Nagoya, Aichi

467-8603, Japan

Full list of author information is available at the end of the article
}

(c) The Author(s). 2018 Open Access This article is distributed under the terms of the Creative Commons Attribution 4.0 International License (http://creativecommons.org/licenses/by/4.0/), which permits unrestricted use, distribution, and reproduction in any medium, provided you give appropriate credit to the original author(s) and the source, provide a link to the Creative Commons license, and indicate if changes were made. The Creative Commons Public Domain Dedication waiver (http://creativecommons.org/publicdomain/zero/1.0/) applies to the data made available in this article, unless otherwise stated. 


\section{Background}

The National Comprehensive Cancer Network clinical practice guidelines recommend systemic cisplatin-based chemotherapy in patients with urothelial carcinoma (UC) who have metastatic or recurrent disease following radical surgery [1]; the gemcitabine (GEM) plus cisplatin regimen is commonly used in clinical practice $[1,2]$. However, because cisplatin is highly protein bound, its acute and chronic toxicity profile is unpredictable in patients undergoing hemodialysis. Therefore, carboplatin (CBDCA), a second-generation platinum agent, is preferred in patients with renal failure because of its predictable kinetics and limited toxicity profile [3]. Some reports have described the use of CBDCA-based chemotherapy in cancer patients undergoing hemodialysis; however, these patients were $<80$ years old [4-9].

For the management of side effects associated with CBDCA, it is important to control the area under the concentration-time curve (AUC) of CBDCA after intravenous administration, because the AUC of CBDCA is correlated with hematologic toxicities, especially nadir thrombocytopenia [10]. Thus, the CBDCA dose should be calculated using Calvert's formula, which incorporates the pretreatment glomerular filtration rates (GFR) along with the AUC of CBDCA [10]. An AUC of $5.0 \mathrm{mg} /$ $\mathrm{mL} \mathrm{min}$ is commonly recommended for patients with locally advanced or metastatic UC [11]. With regards to the administration of CBDCA for hemodialysis patients, prior reports describe the importance of the setting of the CBDCA dose or removal by hemodialysis to control the AUC $[4,9]$. However, standard procedures have not yet been established for CBDCA administration to hemodialysis patients because the administration of anticancer agents to such patients, especially in elderly individuals, is very rare. In addition, no studies have evaluated the quality of life (QOL) of hemodialysis patients receiving CBDCA-based chemotherapy.

We describe the case of an elderly hemodialysis patient with UC who was treated with GEM plus CBDCA chemotherapy by monitoring the measured AUC of CBDCA to determine the optimal dose and hemodialysis timing. Moreover, we also report the results of a QOL survey administered while the patient was receiving chemotherapy.

\section{Case presentation}

The patient was an 81-year-old Japanese man. He was diagnosed with a recurrence of UC with multiple lymph node metastases, originating from the left renal pelvis, 1 year after laparoscopic radical nephroureterectomy. In addition, his renal function worsened 1 month before the above diagnosis; a shunt was created surgically for hemodialysis initiation. Thus, chemotherapy was planned while the patient continued hemodialysis.
Before the commencement of chemotherapy, the patient's Eastern Cooperative Oncology Group performance status was 1 . His height was $163.5 \mathrm{~cm}$, dry weight was $51.90 \mathrm{~kg}$, and body surface area (BSA) was $1.55 \mathrm{~m}^{2}$. Laboratory findings were as follows: white blood count, $3700 / \mu \mathrm{L}$; hemoglobin, $11.0 \mathrm{~g} / \mathrm{dL}$; platelet count, $168 \times$ $10^{3} / \mu \mathrm{L}$; blood urea nitrogen, $24.2 \mathrm{mg} / \mathrm{dL}$; and creatinine, $3.38 \mathrm{mg} / \mathrm{dL}$. The patient had residual renal function, with daily urine volume $>500 \mathrm{~mL}$ and a 24 -h creatinine clearance of $7.3 \mathrm{~mL} / \mathrm{min}$.

The GEM dose was reduced by $25 \%\left(750 \mathrm{mg} / \mathrm{m}^{2}\right)$ and was administered by intravenous infusion for $30 \mathrm{~min}$ on days 1 and 8 of a 21-day cycle. CBDCA was administered by intravenous infusion for $60 \mathrm{~min}$ on day 1 , followed by infusion of GEM. The initial CBDCA dose was calculated according to the Calvert's formula (target AUC: $5.0 \mathrm{mg} / \mathrm{ml} \mathrm{min,} \mathrm{GFR:} 6.1 \mathrm{~mL} / \mathrm{min})$. GFR was calculated based on knowledge that 24-h creatinine clearance is generally approximately $20 \%$ higher than GFR [12]. Therefore, the CBDCA dose was calculated as $160 \mathrm{mg}$.

Hemodialysis commenced $2 \mathrm{~h}$ after the end of CBDCA infusion on day 1 and was performed for $3 \mathrm{~h}$, with a blood flow rate of $200 \mathrm{~mL} / \mathrm{min}$ and a continuous infusion of heparin as an anticoagulant. The dialyzer membrane was made of polymethyl methacrylate, with a surface area of $1.6 \mathrm{~m}^{2}$ (BK1.6P, TORAY Inc., Tokyo, Japan). Subsequent hemodialysis was performed on days 3 and 5.

A pharmacokinetic study was performed to monitor the measured AUC of CBDCA. This study was approved by the ethical review board at Nagoya City University Graduate School of Medical Sciences. Informed consent was obtained from the patient. Blood samples were collected during the first 2 cycles of chemotherapy. Sampling points were as follows: immediately after CBDCA infusion, before starting and ending hemodialysis, and 20 and $48 \mathrm{~h}$ after CBDCA infusion on day 1 (Fig. 1). The plasma was stored at $-80{ }^{\circ} \mathrm{C}$ until analysis. The plasma platinum level was measured by inductively coupled plasma mass spectrometry. The CBDCA level was calculated using the molar ratio of platinum: CBDCA (371.25/195.08). The measured AUC of plasma CBDCA was calculated using the trapezoidal method according to the intervals before, during, and after hemodialysis, with extrapolation to infinity.

The measured AUC of CBDCA in the first cycle was $5.96 \mathrm{mg} / \mathrm{mL}$ min, which was $19.2 \%$ higher than the target AUC (Fig. 1a). Consequently, grade 4 neutropenia and grade 3 thrombocytopenia were observed, according to the National Cancer Institute's Common Toxicity Criteria for Adverse Events version 4.0 (Table 1). To match the target AUC more closely in the subsequent cycle, we performed a pharmacokinetic study using parameters 


\section{a First cycle}

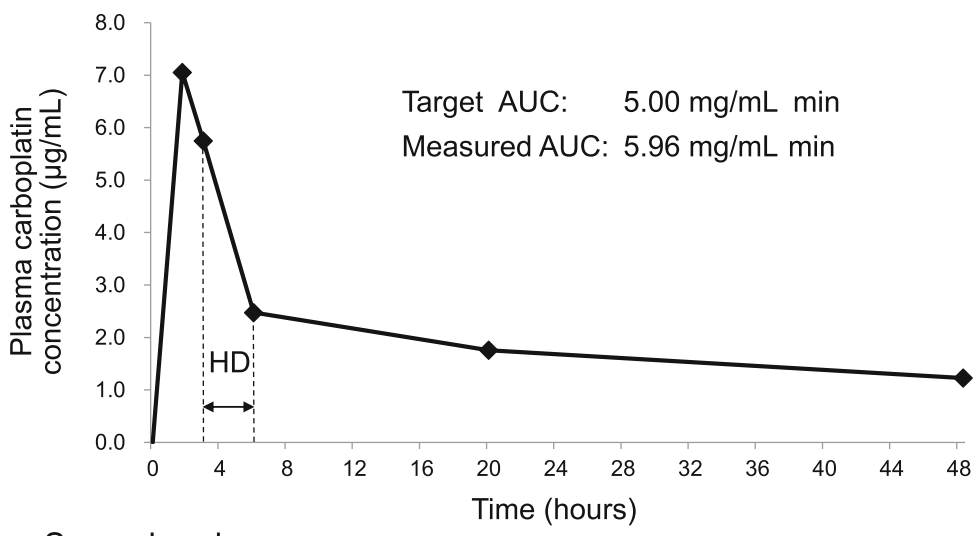

b Second cycle

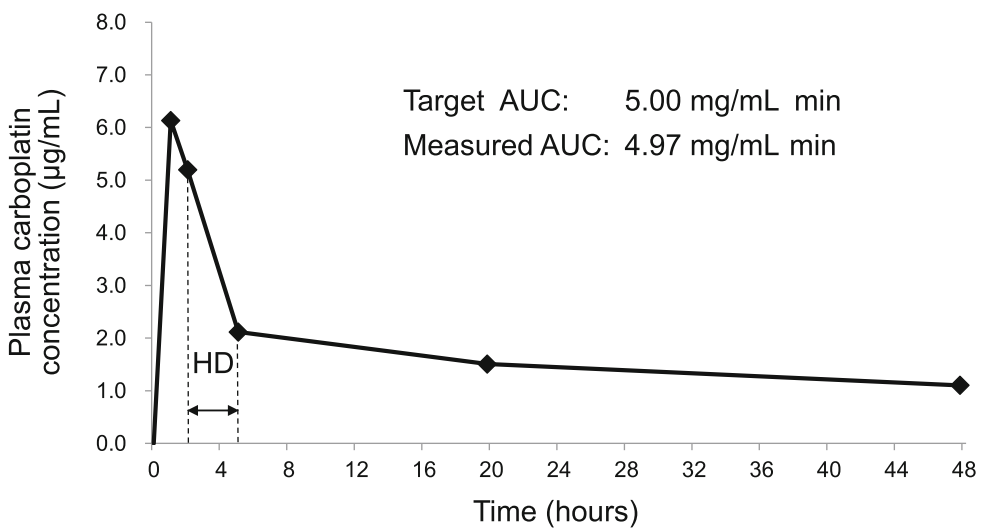

Fig. 1 Plasma concentration-time curves of carboplatin during the (a) first and (b) second chemotherapy cycle. The time intervals between the carboplatin infusion and the commencement of hemodialysis were $2 \mathrm{~h}$ in the first cycle and $1 \mathrm{~h}$ in the second cycle of gemcitabine plus carboplatin chemotherapy. Hemodialysis was performed for $3 \mathrm{~h}$ in each chemotherapy cycle. AUC: area under the concentration-time curve; $H D$, hemodialysis

obtained in the first cycle. Consequently, the CBDCA dose was reduced to $135 \mathrm{mg}$, and the time interval between the CBDCA infusion and hemodialysis initiation was shortened to $1 \mathrm{~h}$ in the second cycle. The hemodialysis duration and conditions, such as the dialyzer and blood or dialysate flow rates, were unchanged. The measured AUC of CBDCA in the second cycle was $4.97 \mathrm{mg} / \mathrm{mL} \mathrm{min}$ (Fig. 1b). The CBDCA dose and administration procedure in the third cycle were the same as that in the second cycle. In the second and third cycles, neutropenia severities were grades 2 and 3 and thrombocytopenia severities were grades 2 and 1 , respectively (Table 1 ). In addition, no other serious adverse events, including nausea and vomiting, were observed by the supportive care. Serum creatinine levels immediately prior to starting the second and third cycles were $3.12 \mathrm{mg} / \mathrm{dL}$ and $2.84 \mathrm{mg} / \mathrm{dL}$, respectively; the 24-h creatinine clearance was only measured prior to the first cycle. The CBDCA removal rates by hemodialysis in the first and second cycles were calculated at 56.9 and 59.3\%, respectively, though the redistribution phenomenon in the post hemodialysis period could not be considered due to the small number of blood sampling

Table 1 The measured AUC of carboplatin and hematologic toxic effects for 3 cycles of chemotherapy

\begin{tabular}{|c|c|c|c|c|c|c|c|c|c|}
\hline \multirow[t]{2}{*}{ Cycle } & \multicolumn{2}{|c|}{ Doses of anticancer agents (mg) } & \multirow{2}{*}{$\begin{array}{l}\text { Measured AUC of CBDCA } \\
(\mathrm{mg} / \mathrm{mL} \mathrm{min})\end{array}$} & \multicolumn{2}{|c|}{ Neutrophil count $(/ \mu \mathrm{L})$} & \multicolumn{2}{|c|}{ Platelet count $\left(\times 10^{3} / \mu \mathrm{L}\right)$} & \multicolumn{2}{|c|}{ Hemoglobin ( $\mathrm{g} / \mathrm{dL})$} \\
\hline & CBDCA & GEM & & Pretreatment & Nadir & Pretreatment & Nadir & Pretreatment & Nadir \\
\hline First & 160 & 1160 & 5.96 & 2100 & 315 & 168 & 40 & 11.0 & 8.2 \\
\hline Second & 135 & 1160 & 4.97 & 5733 & 1248 & 285 & 60 & 11.0 & 9.5 \\
\hline Third & 135 & 1160 & - & 4095 & 800 & 258 & 129 & 9.5 & 8.5 \\
\hline
\end{tabular}

CBDCA carboplatin, GEM gemcitabine, AUC area under the concentration-time curve 
Table 2 Pharmacokinetic parameters of carboplatin in the first and second cycle of gemcitabine plus carboplatin chemotherapy

\begin{tabular}{|c|c|c|c|c|c|c|c|c|}
\hline \multirow[t]{2}{*}{ Treatment phase } & \multicolumn{4}{|l|}{ First cycle } & \multicolumn{4}{|l|}{ Second cycle } \\
\hline & $\begin{array}{l}\text { Measured AUC } \\
\text { (mg/mL min) }\end{array}$ & Ke $\left(h^{-1}\right)$ & $t_{1 / 2}(h)$ & $\mathrm{CL}(\mathrm{mL} / \mathrm{min})$ & $\begin{array}{l}\text { Measured AUC } \\
\text { (mg/mL min) }\end{array}$ & Ke $\left(h^{-1}\right)$ & $t_{1 / 2}(h)$ & $\mathrm{CL}(\mathrm{mL} / \mathrm{min})$ \\
\hline Pre-dialysis & 0.88 & 0.150 & 4.6 & - & 0.47 & 0.211 & 3.3 & - \\
\hline During dialysis & 0.74 & 0.281 & 2.5 & - & 0.66 & 0.300 & 2.3 & - \\
\hline Post-dialysis & 4.34 & 0.017 & 40.8 & - & 3.84 & 0.015 & 46.2 & - \\
\hline Total & 5.96 & _- & _- & 26.8 & 4.97 & - & _- & 27.2 \\
\hline
\end{tabular}

Carboplatin dose in the first and second cycle were $160 \mathrm{mg}$ and $135 \mathrm{mg}$, respectively. The time interval between the carboplatin infusion and hemodialysis initiation in the first and second cycle were two hours and one hour, respectively

$A U C$ area under the concentration-time curve of carboplatin, Ke elimination rate constant, $t_{1 / 2}$ elimination half-life, $C L$ clearance

points. Other pharmacokinetic parameters in the first and second cycles are shown in Table 2. Total clearance of CBDCA was slightly higher in the second cycle than in the first cycle. Stable disease (according to Response Evaluation Criteria in Solid Tumors) was demonstrated by computed tomography after the second and third cycles.

The patient's QOL before treatment and after 2 cycles of treatment was evaluated using the Medical Outcomes Study 36-Item Short Form Survey (SF-36, iHope International Inc., Kyoto, Japan), which is a questionnaire used to measure general health status [13]. Mean norm-based score (NBS) is an international common score recalculated on the basis of the score of 8 items of the SF-36. Our patient's NBS did not significantly decrease after 2 cycles of chemotherapy compared with his NBS before treatment (Table 3).

\section{Discussion and conclusions}

No studies have investigated the safety and the impact on QOL of CBDCA-based chemotherapy in elderly hemodialysis patients $>80$ years old. Herein, we describe the treatment tolerability and effects on QOL of GEM plus CBDCA chemotherapy in such a patient. The initial CBDCA dose, which was calculated using Calvert's

Table 3 The change in mean NBS for QOL before and after 2 cycles of chemotherapy

\begin{tabular}{lll}
\hline Scale name & Mean NBS \\
\cline { 2 - 3 } & Pre-chemotherapy & After 2 cycles \\
\hline Physical functioning & 25.4 & 29.0 \\
Role physical & 52.4 & 49.1 \\
Bodily pain & 66.7 & 66.7 \\
General health perception & 45.8 & 45.8 \\
Vitality & 43.4 & 40.2 \\
Social functioning & 57.0 & 57.0 \\
Role emotional & 51.9 & 47.7 \\
Mental health & 59.8 & 57.2 \\
\hline
\end{tabular}

The patient's QOL was evaluated using the Medical Outcomes Study 36-Item Short Form Survey (SF-36). This survey consisted of 8 items. Mean NBS is an international common score recalculated on the basis of the score on 8 items of the SF-36

NBS norm-based score, $Q O L$ quality of life formula, and hemodialysis timing were determined according to prior reports. However, the measured AUC in the first cycle unexpectedly exceeded the target AUC by about $20 \%$, resulting in the development of grade 4 hematologic toxicities. A prior report concluded that $\mathrm{Cal}-$ vert's formula causes CBDCA overdosing by overestimating CBDCA clearance in adult patients with severe renal insufficiency, including those undergoing hemodialysis, because this formula was originally developed using pharmacokinetic data from Caucasian patients with a GFR ranging from 33 to $135 \mathrm{~mL} / \mathrm{min}[5,10]$. This might explain why the measured AUC exceeded the target AUC in our patient, although this has not been investigated in studies with large numbers of patients.

On the other hand, in a hemodialysis patient, the CBDCA AUC is dependent upon not only its dose, but also the hemodialysis timing. According to some prior reports, hemodialysis timing can be roughly classified as commencing on the day of CBDCA administration (commencement 1 to $2 \mathrm{~h}$ after the end of CBDCA infusion) or commencing on the day after CBDCA administration (commencement 16 to $24 \mathrm{~h}$ after the end of CBDCA infusion) [4-9]. However, the correlation between these 2 hemodialysis timings and the ratio of measured AUC to target AUC has not been established. Moreover, there are also other potential factors which affect the AUC of CBDCA, including the patient's residual renal function and hemodialysis duration and conditions [8]. Various factors in each patient may affect the AUC of CBDCA. Therefore, especially in the first administration of CBDCA, the prediction of the actual AUC following CBDCA infusion is very difficult in patients who are undergoing hemodialysis, although some reports have described that CBDCA-based chemotherapy can be administered to such patients. Therefore, in cases receiving chemotherapy with non-curative intent, similar to our hemodialysis patient, the initial dose of anti-cancer agents in the first cycle should be reduced to take into account the patient's safety and QOL.

When chemotherapy-induced adverse events occur, especially hematologic toxicities, dose reduction is a useful method for decreasing such toxicity, because the AUC of 
CBDCA may be reduced in cases receiving CBDCA-based chemotherapy. However, in hemodialysis patients, it is uncertain whether the AUC of CBDCA and associated toxicities will decrease enough by only standardized dose reduction, as in non-hemodialysis patients, because the AUC of CBDCA is not dependent upon only the dose. Ineffective treatment will result if the dose is reduced too much, although there may be decreased toxicity. Without monitoring the AUC, it would be difficult to discover this insufficient treatment intensity. Therefore, monitoring of the measured AUC following the administration of CBDCA is important as the indicator for setting the appropriate dose or for hemodialysis timing in each hemodialysis patient.

It was previously reported that the total clearance of CBDCA in the second and third cycle decreased to $30.0 \mathrm{~mL} / \mathrm{min}$ and $20.8 \mathrm{~mL} / \mathrm{min}$, respectively, compared to $43.7 \mathrm{~mL} / \mathrm{min}$ in the first cycle, when the CBDCA dose remained unchanged, and the time interval in the second and third cycle was extended from $1 \mathrm{~h}$ to 16 and $20 \mathrm{~h}$, respectively [4]. Thus, the extension of the time interval between CBDCA infusion and hemodialysis initiation could lead to a decrease in the total clearance of CBDCA through the treatment period and then to an increase in the AUC of CBDCA. In our case, we changed both the dose of CBDCA and the hemodialysis timing from the second cycle. The dose was reduced from $160 \mathrm{mg}$ to $135 \mathrm{mg}$, and the time interval between CBDCA infusion and hemodialysis initiation was shortened by $1 \mathrm{~h}$. Consequently, there was a decrease in the measured AUC of CBDCA in the second cycle by about $17 \%$, compared that in to the first cycle. However, the increase in the total clearance in the second cycle was minor compared to that in the first cycle. This may have been due to the extended time interval between CBDCA infusion and the fact that hemodialysis initiation was only $1 \mathrm{~h}$. Thus, in our case, the dose reduction might have more strongly contributed to the decrease in the AUC of CBDCA than shortening the time interval between CBDCA infusion and hemodialysis initiation. However, these results in our study do not suggest that the hemodialysis timing does not contribute to the change in AUC of CBDCA and CBDCA-related side effects, considering previous reports described above [4]. Therefore, monitoring of the measured AUC following the administration of CBDCA is needed, even if there is a short time interval between CBDCA infusion and hemodialysis.

The lack of 24-h creatinine clearance data for the second and third cycle may be a limitation of this study. Following discussion with the physician, we only measured the 24-h creatinine clearance before the first cycle, because the patient's general condition was stable and the patient's burden with urine collection for $24 \mathrm{~h}$ would be reduced for the subsequent chemotherapy cycles. However, renal function should be evaluated before each chemotherapy cycle, because the change in residual renal function may also affect the AUC of CBDCA. Moreover, 24-h creatinine clearance may be a suitable method to evaluate residual renal function for hemodialysis patients with available daily urine volume.

A previous prospective observational study in patients who received chemotherapy, including CBDCA-based regimens, evaluated the impact of chemotherapy-induced neutropenia on QOL [14]. The authors concluded that there may be a QOL decrement associated with development of grade 4 neutropenia. Thus, the adjustment of treatment intensity is needed to avoid decrement in QOL induced by the development of severe hematologic toxicities. Moreover, it is especially important for elderly patients, because older age is commonly recognized as a risk factor for chemotherapy-induced neutropenia and associated complications [15]. In our elderly patient, adverse events in the second and third cycles could be controlled and stable disease was achieved for 3 cycles by the adjustment of CBDCA dose and hemodialysis timing according to the monitoring the measured AUC. Consequently these 2 cycles of chemotherapy did not decrease the patient's QOL.

\section{Conclusions}

CBDCA-based chemotherapy is clinically acceptable and can be considered as a treatment option for hemodialysis patients $>80$ years-old. However, in such patients, it is essential to measure the AUC of CBDCA and to monitor the severity of hematologic toxicities during chemotherapy, because the measured AUC in the first cycle may unexpectedly exceed the target AUC. Moreover, the AUC is hard to predict due to various influencing factors regarding hemodialysis that may be different for each patient. Therefore, further case reports and prospective studies will be needed to assess the optimal dose and administration procedure for CBDCA.

\begin{abstract}
Abbreviations
AUC: Area under the concentration-time curve; BSA: Body surface area; CBDCA: Carboplatin; GEM: Gemcitabine; GFR: Glomerular filtration rate; NBS: Norm-based score; QOL: Quality of life; UC: Urothelial cell carcinoma
\end{abstract}

\section{Acknowledgements}

Not applicable.

\section{Funding}

Not applicable.

\section{Availability of data and materials}

All data generated or analyzed during this study are included in this published article.

\section{Authors' contributions}

MK conceived the study, designed the protocol and drafted the manuscript. MK, TN, and YS monitored the patients and carried out acquisition of the patient data. YY measured the plasma concentration of free platinum. MK, 
NT, YH, TY and KK analyzed and interpreted the data. All authors revised and approved the final manuscript.

\section{Ethics approval and consent to participate}

This study was conducted with the approval of the ethical review board at Nagoya City University Graduate School of Medical Sciences (1176). Written informed consent was obtained from the participant.

\section{Consent for publication}

Written informed consent was obtained from the patient for publication of this case report. A copy of the written consent is available for review by the Editor-in-Chief of this journal.

\section{Competing interests}

The authors declare that they have no competing interests.

\section{Publisher's Note}

Springer Nature remains neutral with regard to jurisdictional claims in published maps and institutional affiliations.

\section{Author details}

'Department of Pharmacy, Nagoya City University Hospital, 1-Kawasumi, Mizuho-cho, Mizuho-ku, Nagoya, Aichi 467-8602, Japan. ${ }^{2}$ Department of Nephro-urology, Nagoya City University Graduate School of Medical Sciences, 1-Kawasumi, Mizuho-cho, Mizuho-ku, Nagoya, Aichi 467-8602, Japan. ${ }^{3}$ Department of Hospital Pharmacy, Graduate School of Pharmaceutical Sciences, Nagoya City University, 3-1, Tanabe dori, Mizuho ku, Nagoya, Aichi 467-8603, Japan. ${ }^{4}$ Aichi Prefectural Institute of Public Health, 7-6, Tsuji machi, Kita ku, Nagoya, Aichi 462-8576, Japan. ${ }^{5}$ Department of Clinical Pharmaceutics, Nagoya City University Graduate School of Medical Sciences, 1-Kawasumi, Mizuho-cho, Mizuho-ku, Nagoya, Aichi 467-8602, Japan.

Received: 22 May 2018 Accepted: 1 November 2018

Published online: 29 November 2018

\section{References}

1. NCCN Clinical Practice Guidelines in Oncology. Bladder Cancer Version 2. 2018. Available from www.nccn.org/professionals/physician_gls/pdf/bladder.pdf. Accessed 15 May 2018.

2. von der Maase H, Hansen SW, Roberts JT, Dogliotti L, Oliver T, Moore MJ, et al. Gemcitabine and cisplatin versus methotrexate, vinblastine, doxorubicin, and cisplatin in advanced or metastatic bladder cancer: results of a large, randomized, multinational, multicenter, phase III study. J Clin Oncol. 2000; 18:3068-77.

3. Motzer RJ, Niedzwiecki D, Isaacs M, Menendez-Botet C, Tong WP Flombaum C, et al. Carboplatin-based chemotherapy with pharmacokinetic analysis for patients with hemodialysis-dependent renal insufficiency. Cancer Chemother Pharmacol. 1990;27:234-8.

4. Wada T, Fukuda T, Kawanishi M, Tasaka R, Imai K, Yamauchi M, et al. Pharmacokinetic analyses of carboplatin in a patient with cancer of the fallopian tubes undergoing hemodialysis: a case report. Biomed Rep. 2016;5: 199-202.

5. Oguri T, Shimokata T, Ito I, Yasuda Y, Sassa N, Nishiyama M, et al. Extension of the Calvert formula to patients with severe renal insufficiency. Cancer Chemother Pharmacol. 2015;76:53-9.

6. Hiraike M, Hiraki Y, Misumi N, Hanada K, Tsuji Y, Kamimura H, et al. Pharmacokinetics of carboplatin in a hemodialysis patient with small-cell lung cancer. Cancer Chemother Pharmacol. 2012;69:845-8.

7. Oguri T, Shimokata T, Inada M, Ito I, Ando Y, Sasaki Y, et al. Pharmacokinetic analysis of carboplatin in patients with cancer who are undergoing hemodialysis. Cancer Chemother Pharmacol. 2010;66:813-7.

8. Kodama J, Sasaki A, Masahiro S, Seki N, Kusumoto T, Nakamura K, et al. Pharmacokinetics of combination chemotherapy with paclitaxel and carboplatin in a patient with advanced epithelial ovarian cancer undergoing hemodialysis. Oncol Lett. 2010;1:511-3.

9. Suzuki S, Koide M, Sakamoto S, Matsuo T. Pharmacokinetics of carboplatin and etoposide in a haemodialysis patient with Merkel-cell carcinoma. Nephrol Dial Transplant. 1997;12:137-40.
10. Calvert AH, Newell DR, Gumbrell LA, O'Reilly S, Burnell M, Boxall FE, et al. Carboplatin dosage: prospective evaluation of a simple formula based on renal function. J Clin Oncol. 1989;7:1748-56.

11. Nogué-Aliguer $\mathrm{M}$, Carles J, Arrivi A, Juan O, Alonso L, Font A, et al. Gemcitabine and carboplatin in advanced transitional cell carcinoma of the urinary tract: an alternative therapy. Cancer. 2003;97:2180-6.

12. Olyaei Aj LEV, Tuchman SA, Sparks MA. Rational dosing of chemotherapy in patients with kidney failure. In: Jhaveri KD, Salahudeen AK, editors. Onconephrology: Cancer, chemotherapy and kidney. New York: Springer; 2015. p. 105-29.

13. Fukuhara S, Bito S, Green J, Hsiao A, Kurokawa K. Translation, adaptation, and validation of the SF-36 health survey for use in Japan. J Clin Epidemiol. 1998;51:1037-44.

14. Fortner BV, Schwartzberg L, Tauer K, Houts AC, Hackett J, Stolshek BS. Impact of chemotherapy-induced neutropenia on quality of life: a prospective pilot investigation. Support Care Cancer. 2005;13:522-8.

15. Lyman GH, Kuderer NM, Crawford J, Wollf DA, Culakova E, Poniewierski MS, et al. Predicting individual risk of neutropenic complications in patients receiving cancer chemotherapy. Cancer. 2011;117:1917-27.

\section{Ready to submit your research? Choose BMC and benefit from:}

- fast, convenient online submission

- thorough peer review by experienced researchers in your field

- rapid publication on acceptance

- support for research data, including large and complex data types

- gold Open Access which fosters wider collaboration and increased citations

- maximum visibility for your research: over $100 \mathrm{M}$ website views per year

At $\mathrm{BMC}$, research is always in progress.

Learn more biomedcentral.com/submissions 